\title{
Collapsing multiway contingency tables: Simpson's paradox and homogenization
}

\author{
JOHN R. VOKEY \\ University of Lethbridge, Lethbridge, Alberta, Canada
}

\begin{abstract}
Simpson's paradox results from the collapsing of multiway contingency tables in the presence of nonadditivity. An extension of the iterative proportional fitting algorithm (Deming \& Stephan, 1940) is described that may be used to homogenize such tables to produce the required additivity over the unwanted (collapsed-over) effects.
\end{abstract}

Collapsing multiway contingency tables in the presence of nonadditivity results in what Blyth (1972) called Simpson's paradox, following an earlier discussion of the phenomenon by Simpson (1951): a reversal of relationship across different levels of stratification, resulting from heterogeneity in the dimension or dimensions collapsed over to produce the aggregate table. More recently (e.g., Hintzman, 1980), the term has been generalized to include changes in magnitude in addition to changes in sign of relationship following the collapsing of contingency tables, such as, for example, where an apparent relationship emerges in the aggregated table even though none existed in the individual tables collapsed over. The purpose of this paper is to demonstrate a simple, general method of homogenizing contingency tables to produce the required additivity over the unwanted (i.e., collapsedover) effects in a manner analogous to partial correlation or statistical control by analysis of covariance.

That Simpson's paradox can and does occur is well established. Note of at least some aspects of the general problem associated with collapsing over heterogeneity dates back at least as far as Yule (1903), and real-world examples of the reversal paradox were noted as early as M. R. Cohen and Nagel (1934). More recent real-world examples may be found in Knapp (1985), Mantell (1982), and Wagner (1982). Further discussions and conceptualizations of the issue may be found in J. E. Cohen (1986), Flexser (1981), Gardner (1976), Hintzman (1980), Martin (1981), Nyberg (1993), Paik (1985), Shapiro (1982), Sunder (1983), and Wickens (1993). Haunsberger and Saari (1991), Saari (1987), and Simon and Blume (1994, chap. 15) present a more general conceptualization of the problem. The problem is not limited to the collapsing of contingency tables; Simpson's paradox is but a special case of the more general problem of cross-level inferences

This work was supported by an operating grant from the National Sciences and Engineering Research Council of Canada to the author. The author thanks Gil Becker for introducing him to the problem. Program listings and software to perform homogenization are available from the author. Correspondence should be addressed to J. R. Vokey, Department of Psychology, University of Lethbridge, Lethbridge, AB, Canada T1K3M4 (e-mail: vokey@hg.uleth.ca). (e.g., Knapp, 1977; Lindquist, 1940; Robinson, 1950; Thorndike, 1939), although it is not often portrayed as such (but see Paik, 1985; Wermuth, 1989). The "paradox" in Simpson's paradox, or cross-level inferences more generally, arises from the typically unwarranted assumption or expectation that aggregates should evince the same relationships as the categories, levels, classes, items, or individuals over which the aggregate was formed.

Probably the best known real-world example of Simpson's paradox is that provided by Bickel, Hammel, and O'Connell (1975) concerning an apparent gender bias in acceptances to graduate school at Berkeley in 1973. Although for the 101 departments as a whole, males were accepted proportionately more often than were females, the data for the individual departments suggested that, if any, there was a bias in favor of accepting female students. The frequency data relating gender of applicant to graduate school acceptance for each of the six largest departments are shown in Table $1 \mathrm{~A}$, and the marginal table and corresponding proportions relating gender of applicant to acceptance, collapsed across department, are shown as the last two columns. The reversal phenomenon is evident in this reduced data set, and the explanation for the apparent paradox here is the same as that reported by Bickel et al. (1975) for the complete set of departments: Although, generally, within departments, female applicants were proportionately as likely as (if not more likely than) male applicants to be accepted, females had a proportionately lower overall rate of acceptance because they applied disproportionately more often to the departments with the lowest rates of acceptance.

A more recent example is given by Wardrop (1995), who argued that the "hot hand" in basketball often claimed by aficionados of the sport is consistent with the (presumably aggregate) data available to them (in this specific case, free-throw data collapsed over 9 shooters from the 1980-1982 Boston Celtics), despite Tversky and Gilovich's (1989) report of no evidence (on average) for the claim at the level of individual shooters (i.e., 4 players shot better after a hit, and 5 players shot better after a miss). Here, as noted by Gilovich, Vallone, and Tversky (1985), who warned against such collapsing, the explanation for the apparent paradox is that good shooters contribute dis- 
Table 1

Frequencies of Male and Female Applicants Accepted or Rejected for Graduate School in the Six Largest Departments at Berkeley in 1973

\begin{tabular}{|c|c|c|c|c|c|c|c|c|c|c|}
\hline \multirow[b]{2}{*}{ Subtable } & \multirow[b]{2}{*}{ Gender } & & \multicolumn{6}{|c|}{ Department } & \multirow[b]{2}{*}{ Total } & \multirow[b]{2}{*}{$\%$} \\
\hline & & & A & B & $\mathrm{C}$ & D & E & $\mathrm{F}$ & & \\
\hline \multirow[t]{5}{*}{ A } & Male & Accept & 512 & 353 & 120 & 139 & 53 & 22 & 1,199 & 45 \\
\hline & & Reject & 313 & 207 & 205 & 278 & 138 & 351 & 1,492 & 55 \\
\hline & Female & Accept & 89 & 17 & 202 & 131 & 94 & 24 & 557 & 30 \\
\hline & & Reject & 19 & 8 & 391 & 244 & 299 & 317 & 1,278 & 70 \\
\hline & & Total & 933 & 585 & 918 & 792 & 584 & 714 & 4,526 & \\
\hline \multirow[t]{5}{*}{ B } & Male & Accept & 453.6 & 529.3 & 96.9 & 142.1 & 71.0 & 30.2 & 1,323 & 58 \\
\hline & & Reject & 152.9 & 171.2 & 91.3 & 156.7 & 101.9 & 265.9 & 940 & 42 \\
\hline & Female & Accept & 132.3 & 42.8 & 273.8 & 224.7 & 211.1 & 55.3 & 940 & 42 \\
\hline & & Reject & 15.6 & 11.1 & 292.3 & 230.8 & 370.4 & 402.9 & 1,323 & 58 \\
\hline & & Total & 754 & 754 & 754 & 754 & 754 & 754 & 4,526 & \\
\hline \multirow[t]{5}{*}{ C } & Male & Accept & 159.3 & 127.6 & 218.3 & 179.5 & 141.4 & 157 & 983 & 37 \\
\hline & & Reject & 395.4 & 220.3 & 327.5 & 291.4 & 205.9 & 267.6 & 1,708 & 63 \\
\hline & Female & Accept & 202.7 & 99.4 & 137.9 & 127.8 & 85.2 & 120.1 & 773 & 42 \\
\hline & & Reject & 175.6 & 137.8 & 234.3 & 193.3 & 151.6 & 169.4 & 1,062 & 58 \\
\hline & & Total & 933 & 585 & 918 & 792 & 584 & 714 & 4,526 & \\
\hline
\end{tabular}

Note-Subtable A presents the original data reconstructed from the corresponding proportions reported by Freedman, Pisani, and Purves (1978). Subtable B presents the frequencies adjusted to reflect uniform main marginals. Subtable $C$ presents the frequencies homogenized for the effects of department $X$ gender and department $\times$ acceptance, maintaining the original main marginal totals.

proportionately but not contingently to hits, rather than misses, preceded by hits, whereas poor shooters contribute in the opposite way, thereby producing the effect that, collapsed over shooters, hits disproportionately follow hits. The frequency data relating first- and secondshot hits and misses as a function of shooter are shown in Table $2 \mathrm{~A}$, and the marginal table and corresponding proportions relating second-shot hits and misses to firstshot performance, collapsed across shooter, are shown as the last two columns.

Pooling individual tables (typically subjects or observational units) or collapsing across one or more variables in complex tables is a commonplace operation with discrete, cross-classified data. Occasionally, the interest is, in fact, in the distribution over or the relationships among the remaining variables at the class, category, or group levels of the resulting table; if so, the possibility of Simpson's paradox (i.e., that lower level aggregates of the same variables could potentially evince different relationships) is not an issue. More commonly, though, the intent is to produce a simpler table that preserves the distributions or relationships inherent in the collapsed-over tables or strata. Discussion of the necessary and sufficient conditions for such collapsibility (which vary as a function of, inter alia, the measure of association used and whether sign or both sign and magnitude are to be preserved) may be found in Bishop, Fienberg, and Holland (1975), Shapiro (1982), and Whittemore (1978). For present purposes, it is necessary to recognize only that collapsibility is possible if the collapsed-over variables or strata are marginally independent of the variables entering into the relationships of interest - that is,

Table 2

First- and Second-Shot Free-Throw Data of 9 Shooters From the Boston Celtics During the $1980-1982$ Seasons

\begin{tabular}{|c|c|c|c|c|c|c|c|c|c|c|c|c|c|}
\hline \multirow[b]{2}{*}{ Subtable } & \multicolumn{2}{|c|}{ Shot } & \multicolumn{9}{|c|}{ Shooter } & \multirow[b]{2}{*}{ Total } & \multirow[b]{2}{*}{$\%$} \\
\hline & First & Second & L.B. & C.M. & R.P. & N.A. & C.F. & K.M. & M.C. & R.R. & G.H. & & \\
\hline \multirow[t]{5}{*}{ A } & Hit & Hit & 251 & 245 & 164 & 203 & 36 & 93 & 39 & 54 & 77 & 1,162 & 79 \\
\hline & & Miss & 34 & 57 & 49 & 42 & 15 & 35 & 18 & 37 & 24 & 311 & 21 \\
\hline & Miss & Hit & 48 & 97 & 76 & 62 & 17 & 29 & 21 & 49 & 29 & 428 & 74 \\
\hline & & Miss & 5 & 31 & 29 & 14 & 5 & 20 & 5 & 31 & 8 & 148 & 26 \\
\hline & & Total & 338 & 430 & 318 & 321 & 73 & 177 & 83 & 171 & 138 & 2,049 & \\
\hline \multirow[t]{5}{*}{ B } & Hit & Hit & 110.5 & 61.2 & 50.7 & 77.0 & 49.6 & 48.8 & 47.3 & 22.9 & 60.8 & 529 & 52 \\
\hline & & Miss & 47.6 & 45.3 & 48.2 & 50.7 & 65.8 & 58.5 & 69.5 & 49.9 & 60.3 & 496 & 48 \\
\hline & Miss & Hit & 52.3 & 60.0 & 58.2 & 58.2 & 58.0 & 37.7 & 63.1 & 51.4 & 56.7 & 496 & 48 \\
\hline & & Miss & 17.3 & 61.0 & 70.6 & 41.8 & 54.3 & 82.7 & 47.8 & 103.5 & 49.8 & 529 & 52 \\
\hline & & Total & 227.7 & 227.7 & 227.7 & 227.7 & 227.7 & 227.7 & 227.7 & 227.7 & 227.7 & 2,049 & \\
\hline \multirow[t]{5}{*}{$\mathrm{C}$} & Hit & Hit & 185.5 & 244.8 & 180.2 & 180.1 & 39.9 & 102.7 & 44.5 & 94.9 & 76.4 & 1,149 & 78 \\
\hline & & Miss & 57.4 & 64.3 & 48.4 & 50.7 & 12.6 & 24.5 & 15.1 & 28.0 & 22.8 & 324 & 22 \\
\hline & Miss & Hit & 76.7 & 88.8 & 66.6 & 69.0 & 16.8 & 34.6 & 19.9 & 37.8 & 30.7 & 441 & 77 \\
\hline & & Miss & 18.3 & 32.0 & 22.8 & 21.2 & 3.7 & 15.1 & 3.5 & 10.3 & 8.1 & 135 & 23 \\
\hline & & Total & 338 & 430 & 318 & 321 & 73 & 177 & 83 & 171 & 138 & 2,049 & \\
\hline
\end{tabular}

Note-Subtable A presents the original frequencies reconstructed from proportions reported by Gilovich, Vallone, and Tversky (1985). Subtable B presents the frequencies adjusted to reflect uniform main marginals. Subtable $C$ presents the frequencies homogenized for the effects of shooter $\times$ first shot and shooter $\times$ second shot, maintaining the original main marginal totals. 
independent of each of these latter variables in the marginal tables formed by collapsing over the remaining variables of interest. Thus, if some to-be-collapsed table can be adjusted to achieve such marginal independence, or homogeneity, then the homogenized table may be collapsed with impunity. Flexser (1981) describes a procedure for homogenizing $2 \times 2$ contingency tables obtained by collapsing over subjects and items for the individual effects of these two sources. The procedure described here, based on the Deming and Stephan (1940) iterative proportional fitting algorithm, is more general, providing for the adjustment of multiway tables to achieve the desired homogeneity over any effects, simple or complex. The algorithm in generalized form is developed first, followed by a discussion of some of its more common uses. Two methods of homogenization are then presented as direct extensions of these procedures, followed by a brief discussion of extensions and limitations of the technique.

\section{The Generalized Deming-Stephan Algorithm}

The Deming and Stephan (1940) iterative proportional fitting procedure may be generalized as the following iterative expression for estimating from $V(V \geq 1 ; v=1$, $\ldots, V)$ marginal effects the value of each cell in a $K$-way contingency table. Suppose that the frequency in cell $(i$, $j, \ldots, k)$ of this table is $m_{i j \ldots k}$, such that $\sum_{i j \ldots k} m_{i j \ldots k}=$ $N, m_{i j+} \ldots$ is a marginal value in one of the $\left(2^{\mathrm{K}}-1\right)$ possible marginal tables (where the plus [ + ] sign conventionally denotes a collapsed-over variable or way), and $n(n=0$, $1,2, \ldots)$ is the current major iteration for the $V$ effects, then

$$
\hat{m}_{i j \ldots k}^{[n V+v]}=\hat{m}_{i j \ldots k}^{[n V+\nu-1]} \frac{E_{i j+\ldots}}{\hat{m}_{i j+\ldots}^{[n V+\nu-1]}},
$$

where $E_{i j+\ldots}$ is the desired (fixed or known) marginal for a given effect, $\hat{m}_{i j+\ldots}^{[n V+\nu-1]}$ is the corresponding marginal from iteration $[n V+v-1]$, and all effects $v$ are cycled through for each major iteration $n$. Iteration continues until convergence-that is, until

$$
\max \left|\hat{m}_{i j \ldots k}^{[n V]}-\hat{m}_{i j \ldots k}^{[(n-1) V]}\right| \leq e,
$$

where $e(e \geq 0)$ is some arbitrary small value (e.g., 0.001 ). The fitting of effects is hierarchical; for a threeway table, for example, fitting effect $E_{i j+}$ also fits effects $E_{i++}$ and $E_{+j+}$.

\section{Computing Expected Values}

The most common use of the procedure is the fitting of models-consisting of different sets of marginal effects - to frequency data. Descriptions of this use of the iterative proportional fitting algorithm may be found in many sources (e.g., Bishop et al., 1975; Fienberg, 1970, 1977; Goodman, 1970; Kennedy, 1983). Haberman (1972) provides a FORTRAN listing of this version of the algorithm for multiway tables, which in, turn serves as the underlying basis for the $\log$-linear analysis of fre- quency data in various statistical computer programs (e.g., BMDP4F of the BMDP statistical software series [Brown, 1983], and the TABLES module of the SYSTAT package [Wilkinson, 1987]). The immediate concern of each of these descriptions (or programs) is the production of a table of expected values, which is then compared with the original table of frequencies either to provide some minimal set of effects that reproduce the data with no significant residual or to enable the investigation of the residual associations themselves. The distinguishing characteristics of this variant of the algorithm are that the cell estimates are initialized to a constant, typically 1.0 (i.e., a state of complete marginal independence), and the values of $E_{i j}+\ldots$ are the values for the corresponding marginal tables from the original data.

\section{Adjusting tu Known or Desired Marginals}

Although the computation of expected values represents the bulk of its current use, the Deming-Stephan (1940) algorithm was proposed originally as a method of adjusting tables to reflect or incorporate specific marginal totals while maintaining the pattern of relationships inherent in the original data. The distinguishing characteristics of this variant of the procedure are that the original cell frequencies are used as the initial cell estimates, and the $E_{i j}+\ldots$ are typically arbitrary (although necessarily mutually compatible) marginal table frequencies. Deming (1943), for example, demonstrated how the procedure may be used to adjust frequency tables obtained from samples so that one or more of the marginals (i.e., $E_{i j}+\ldots$ ) can be made to correspond to those known for the population as a whole, such that the resulting adjusted cell frequencies can serve as maximumlikelihood estimates of unknown, difficult, or impossibleto-obtain population frequencies (see also Haberman, 1979). Mosteller (1968) demonstrated, inter alia, how the procedure could be used to make patterns in contingency tables more apparent by adjusting the cell frequencies to sum to arbitrary but more convenient marginal totals.

As an example of this latter operation, consider adjusting the data in Table 1 so that every department has the same number of applicants, that there are as many male applicants as female applicants, and that as many students are accepted in total as are rejected. Although any positive value would do as the total frequency, we will use the original $N$ and simply partition it equally among the marginal cells of each simple marginal table. The results of iterating equation 1 with these values are shown in Table 1B. Adjusting the data in Table 2 via Equation 1 so that every shooter has the same number shots, that there are as many first-shot hits as misses, and that there are as many second-shot hits as misses results in the values shown in Table 2B. Note first how the pattern of relationships is maintained in the new tables, despite the now-uniform distributions over the main marginals, and note that, in particular, the "paradox" in each 
data set is also unaffected. Note further how this adjustment of the data rendered the marginal association between gender and acceptance or first shots and second shots more readily apparent.

\section{Homogenizing for Effects}

The procedure to homogenize contingency tables for one or more marginal effects is a direct extension of the Deming-Stephan procedure for adjusting tables to known or desired marginals. As discussed, the goal is to adjust the table of frequencies to achieve additivity and, hence, collapsibility over these effects. Again, the original cell frequencies are used as the initial cell estimates. The $E_{i j+\ldots}$, however, instead of being arbitrary, are chosen so as to provide the required marginal independence. There are two ways in which this may be done: one that provides for uniform distributions over the main marginals of the effects to be homogenized (thereby facilitating subsequent interpretation), and one that preserves the main marginals of the original data. For the simpler case of uniform marginals, the $E_{i j+\ldots}$ for each effect are computed as the mean cell frequency for the effect in question. That is, where $c$ is the number of cells in the marginal table for a given effect, $E_{i j+\ldots}=N / c$.

Maintaining the main marginals is slightly more complicated, and it requires that the marginal independence of the $E_{i j+\ldots}$ for each effect be computed from the generalization of the method for computing expected values (i.e., values of marginal independence) for two-way tables. That is, where $W(w=1,2, \ldots, W)$ is the number of ways (variables) in a given marginal table or effect, and $m_{w}=\left\{m_{i+\ldots}, m_{+j+\ldots}, m_{++k+\ldots}, \ldots\right\}$, the marginal values from the original data for each variable defining the cell in question, then

$$
E_{i j+\ldots}=\frac{\prod_{w} m_{w}}{N^{W-1}} .
$$

Consider again the data in Table 1. To collapse these data over the variable of department to produce a simple marginal table relating gender to acceptance that correctly summarizes the relationship at the department level requires that the table be adjusted or homogenized for the effects of department $\times$ gender and the effects of department $\times$ acceptance. ${ }^{l}$ That is, we wish to produce a table from which these marginal associations have been removed and in which the partial associations of gender $x$ acceptance and of gender $\times$ acceptance $X$ department have been retained. Stated differently, in a manner analogous to statistical control by analysis of covariance, we want a table in which departments have been equated both for the rate of acceptance and for the relative number of females that apply. Similarly, for the data in Table 2, we want a table in which the shooters have been equated for both first-shot and second-shot hit rates, so that the table, now homogenized for the effects of shooter $X$ first shot and the effects of shooter $\times$ second shot, when collapsed over shooters, correctly summarizes the rela- tionship at the shooter level. The results obtained by homogenization, preserving the main marginals, are shown in Tables $1 \mathrm{C}$ and $2 \mathrm{C}$. In both cases, the table produced by collapsing now demonstrates the unweighted mean association between the two critical variables at the level of the collapsed-over departments or shooters and shows that the "paradox" in each case has been eliminated.

Collapsing across more than a single variable is handled in the same way. For example, suppose that, for a four-way $(I, J, K$, and $L)$ contingency table, the homogenized table of interest is $K \times L$. Using the iterative proportional procedure just described to homogenize for the effects $I \times J \times K$ and $I \times J \times L$ will produce a table containing only the residual effects of $K \times L, I \times K \times L, J \times$ $K \times L$, and $I \times J \times K \times L$, reflective of the unweighted average of these effects at the level of the individual $I \times J$ units.

\section{Discussion}

Although the core equation can be seen to be the same, the result of the Deming-Stephan iteration procedure used to homogenize for effects is, in many respects, the opposite of that used to fit (i.e., compute expected values from) the same effects. That is, the computation of expected values via the Deming-Stephan procedure results in a table that includes only the effects (including lower order components) specified, whereas homogenization by the same basic process results in a table from which the same effects have been removed. Alternatively, the computation of expected values may be thought of as a procedure by which effects unrelated to and of higher order than those fit are removed from a table, and the homogenization procedure may be thought of as one in which only these unrelated and higher order effects are retained. The homogenization technique may be used to remove effects from a table, whether the intent is to collapse the table subsequently (in which case the variables to be collapsed over dictate the effects to be homogenized) or merely to produce a table for which particular effects have been controlled.

These two uses of the Deming-Stephan procedure differ in other ways as well. Although both will tolerate sampling zeros in the original data as long as a small positive constant is added to every cell to satisfy the mathematics, only the fitting algorithm can also handle structural zeros-cells empty by definition-simply by ignoring such cells in the computation of each of the terms in Equation 1 (e.g., see Haberman, 1972). The problem for the homogenization algorithm is that structural zeros may result in incompatible (nonzero) marginal values to be fitted, with the result that Equation 1 never converges.

On the other hand, the fitting of effects and homogenizing for them can be seen to be complementary, in the same way that the test of effects in analysis of covariance is complementary to the table of adjusted means depicting them. One common method for handling problems of Simpson's paradox arising from collapsing over subjects 
or observational units is to compare the fit of models conditional on the collapsed-over units (see, e.g., Wickens, 1989). Taking the graduate school data in Table 1 as an example (where $\mathrm{G}=$ gender, $\mathrm{A}=$ acceptance, and $\mathrm{D}=$ department), one could compute the difference in fit, $\Delta \mathrm{G}^{2}{ }_{\mathrm{GA}}$ (where $\Delta \mathrm{G}^{2}{ }_{\mathrm{GA}}$ is the likelihood-ratio chi square), between the model [GD] [AD] and the model [GD] [AD] [GA] to test for an effect of gender $\times$ acceptance, conditional on the effects of gender $\times$ department and acceptance $\times$ department (see Wickens, 1993, for a discussion of this method and other methods of analyzing contingency tables with between-unit heterogeneity). The collapsed table corresponding to this conditional tested effect of gender $\times$ acceptance is the homogenized table shown in the marginal of Table 1C.

\section{Program Availability}

A program to perform all three applications of the iterative proportional fitting algorithm to multiway frequency tables is available as a HyperCard stack or as standalone application for Macintosh computers. The program includes the two example data sets discussed in this article and a data set from Radelet (1981) that relates death penalty verdict to defendant's and victim's race. Because all of the computer code is written in $\mathrm{Hy}$ perTalk (Apple computer's object-based scripting language with an English-like syntax), it is readily translated to other computer languages and systems. For users of these other languages or systems, a source-code listing is available. Both the software and the code listings are available from the author's Web page (http://www. uleth.ca/ vokey), or they may be obtained by contacting the author.

\section{REFERENCES}

Bickel, P. J., Hammel, E. A., \& O'Connell, J. W. (1975). Sex bias in graduate admissions: Data from Berkeley. Science, 187, 398-404.

Bishop, Y. M. M., Fienberg, S. E., \& Holland, P. W. (1975). Discrete multivariate analysis: Theory and practice. Cambridge, MA: MIT Press.

Blyth, C. R. (1972). On Simpson's paradox and the sure thing principle. Journal of the American Statistical Association, 67, 364-381.

Brown, M. B. (1983). P4F: Two-way and multiway frequency tablesMeasures of association and the log-linear model (complete and incomplete tables). In BMDP statistical software [Computer software]. Berkeley, CA: University of California Press.

COHEN, J. E. (1986). An uncertainty principle in demography and the unisex issue. American Statistician, 41, 32-39.

COHEN, M. R., \& NAGEL, E. (1934). An introduction to logic and scientific method. New York: Harcourt, Brace \& World.

DEMING, W. E. (1943). Statistical adjustment of data. New York: Dover.

Deming, W. E., \& Stephan, F. F. (1940). On a least squares adjustment of a sampled frequency table when the expected marginal totals are known. Annals of Mathematical Statistics, 11, 427-444.

FIENBERG, S. E. (1970). An iterative procedure for estimation in contingency tables. Annals of Mathematical Statistics, 41, 907-917.

FIENBERG, S. E. (1977). The analysis of cross-classified categorical data. Cambridge, MA: MIT Press.

FLEXSER, A. J. (1981). Homogenizing the $2 \times 2$ contingency table: A method for removing dependencies due to subject and item differences. Psychological Review, 88, 327-339.
Freedman, D., Pisani, R., \& Purves, R. (1978). Statistics. New York: Norton.

GARDNER, M. (1976). On the fabric of inductive logic and some probability paradoxes. Scientific American, 234, 119-124.

Gilovich, T., Vallone, R., \& Tversky, A. (1985). The hot hand in basketball: On the misperception of random sequences. Cognitive Psychology, 17, 295-314.

GoODMAN, L. A. (1970). The multivariate analysis of qualitative data: Interactions among multiple classifications. Journal of the American Statistical Association, 65, 226-256.

Haberman, S. J. (1972). Algorithm AS 51. Log-linear fit for contingency tables. Applied Statistics, 21, 218-225.

HABERMAN, S. J. (1979). Analysis of quantitative data: Volume 2-New developments. New York: Academic Press.

HAUNSBERGER, D., \& SAARI, D. G. (1991). The lack of consistency for statistical decision procedures. American Statistician, 45, 252255.

Hintzman, D. L. (1980). Simpson's paradox and the analysis of memory retrieval. Psychological Review, 87, 398-410.

KENNEDY, J. J. (1983). Analyzing qualitative data: Introductory log-linear analysis for behavioral research. New York: Praeger.

KNAPP, T. R. (1977). The unit-of-analysis problem in applications of simple correlation analysis to educational research. Journal of Educational Statistics, 2, 171-186.

KNAPP, T. R. (1985). Instances of Simpson's paradox. College Mathematics Journal, 16, 209-211.

LINDQUIST, E. F. (1940). Statistical analysis in educational research. Boston: Houghton Mifflin.

Mantell, N. (1982). Simpson's paradox in reverse. American Statistician, 36, 395.

MarTIN, E. (1981). Simpson's paradox resolved: A reply to Hintzman. Psychological Review, 88, 372-374.

Mosteller, F. (1968). Association and estimation in contingency tables. Journal of the American Statistical Association, 63, 1-28.

NyBERG, L. (1993). More on Simpson's paradox and the analysis of memory retrieval. Bulletin of the Psychonomic Society, 31, 326-328.

PaIK, M. (1985). A graphic representation of a three-way contingency table: Simpson's paradox and correlation. American Statistician, 39, 53-54.

RADELET, M. (1981). Racial characteristics and the imposition of the death penalty. American Sociology Review, 46, 918-927.

RoBinson, W. S. (1950). Ecological correlations and the behavior of individuals. American Sociological Review, 15, 351-357.

SAARI, D. G. (1987). The source of some paradoxes from social choice and probability. Journal of Economic Theory, 41, 1-22.

ShAPIRo, S. H. (1982). Collapsing contingency tables-A geometric approach. American Statistician, 36, 43-46.

Simon, C. P., \& Blume, L. (1994). Mathematics for economists. New York: Norton.

SimpSON, E. H. (1951). The interpretation of interaction in contingency tables. American Statistician, 13, 238-241.

SUNDER, S. (1983). Simpson's reversal paradox and cost allocation. Journal of Accounting Research, 21, 222-233.

THORNDIKE, E. L. (1939). On the fallacy of imputing the correlations found for groups to individuals or smaller groups composing them. American Journal of Psychology, 52, 122-124.

TVersky, A., \& Gllovich, T. (1989). The cold facts about the "hot hand" in basketball. CHANCE: New Directions for Statistics \& Computing, 2, 16-21.

WAGNER, C. H. (1982). Simpson's paradox in real life. American Statistician, 36, 46-48.

WARDROP, R. L. (1995). Simpson's paradox and the hot hand in basketball. American Statistician, 49, 24-29.

WERMUTH, N. (1989). Moderating effects of subgroups in linear models. Biometrika, 76, 81-92.

WhitTEMORE, A. S. (1978). Collapsibility of multi-dimensional contingency tables. Journal of the Royal Statistical Society: Series B, 40, 328-340.

WiCKENS, T. D. (1989). Multiway contingency tables analysis for the social sciences. Hillsdale, $\mathrm{NJ}$ : Erlbaum. 
WICKENS, T. D. (1993). Analysis of contingency tables with betweensubjects variability. Psychological Bulletin, 113, 191-204.

WILKINSON, L. (1987). SYSTAT: The system for statistics. Evanston, IL: SYSTAT.

YULE, G. U. (1903). Notes on the theory of association of attributes in statistics. Biometrica, 2, 121-134.

\section{NOTE}

1. Technically, to control for the reversal problem in the collapsed table, it is necessary to homogenize only for one or the other of these two effects, producing the "semi-partial" table. To also control for changes in magnitude, however, both effects must be homogenized. For example, homogenizing only for department $\times$ gender (i.e., equating application rates of the two genders) results in overall acceptance rates of .388 for males and .430 for females (cf. Freedman et al. 1978); homogenizing only for department $\times$ acceptance (i.e., equating departmental acceptance rates) results in acceptance rates of .381 for males and .399 for females. As is shown in the last column of Table $1 \mathrm{C}$, homogenizing for both effects results in acceptance rates of 365 for males and .421 for females. As with the computation of fitted tables, if the table is to be homogenized for only one effect (or the multiple effects are independent), iteration and, hence, the homogenization algorithm are not necessary (although the same result will be produced) because a directly computable solution is available. Freedman et al. (1978), for example, computed their adjusted rates (the weighted average admission rate for each gender, adjusting for the different departmental application rates of the males and females) through weighting the departmental acceptance rates for each gender by the total number of applicants (male and female) to each department, which is identical to what occurs with the homogenization algorithm, maintaining the original main marginals of department and gender. Another approach, suggested by a reviewer, would be to compute the unweighted averages (over department) of the acceptance rates of each gender. In this case, the acceptance rates of .382 for males and .417 for females are equivalent to homogenizing for department $\times$ gender without maintaining the original marginals (i.e., producing uniform main marginals for department and gender).

(Manuscript received October 2, 1996; revision accepted for publication December 17, 1996.) 